\title{
Editorial
}

\section{Active and Passive Microwave Devices and Circuits}

\author{
T. S. Kalkur, ${ }^{1}$ Tibor Berceli, $^{2}$ and Fahrettin Yakuphanoglu ${ }^{3}$ \\ ${ }^{1}$ Department of Electrical and Computer Engineering, University of Colorado, Colorado Springs, CO 80933, USA \\ ${ }^{2}$ Broadband Infocommunications and Electromagnetics Department, Budapest University of Technology and Economics, \\ Goldmann Gyorgy ter 3, 1111 Budapest, Hungary \\ ${ }^{3}$ Physics Department, Faculty of Arts and Sciences, Firat University, 23119, Elazig, Turkey
}

Correspondence should be addressed to T. S. Kalkur, kalkur@eas.uccs.edu

Received 18 August 2008; Accepted 18 August 2008

Copyright (c) 2008 T. S. Kalkur et al. This is an open access article distributed under the Creative Commons Attribution License, which permits unrestricted use, distribution, and reproduction in any medium, provided the original work is properly cited.

New systems working at RF, microwave, and millimeter wave will give rise to new services for medical, security, home, entertain, and communication industries. Active and Passive Microwave Devices and Circuits are playing an important role in the design of these systems. This special issue of Active and Passive components is devoted towards the design, analysis, and fabrication of Active and Passive components.

In the first paper entitled "Dependence on frequency of the electromagnetic field distribution inside a cylindrical cavity," the authors explain the relevant changes in the electron cyclotron resonance ion source behavior for small variations of the exciting radiation frequency.

The second paper in this issue "Printed antenna arrays with side lobe suppression" investigates approaches and their limitations to minimize side lobes in printed antenna arrays. It also proposes antenna structures with printed pentagonal dipole arrays.

The third paper "Isotropic broad-band E-field probe" discusses the development and characterization of isotropic broad-band E-field probe which has an isotropic reception from $100 \mathrm{MHz}$ to $18 \mathrm{GHz}$.

In satellite communication networks, the noise performance and the required spectral purity of the low noise band receiver are of the important issues.

The fourth paper "Ka band phase locked loop oscillator for satellite EHF band receiver" deals with the design and fabrication of Ka band phase locked loop oscillator (PLL DRO) with a fundamental frequency $19.250 \mathrm{GHz}$ for satellite EHF band receiver.

The fifth paper "The novel microwave stop-band filter" proposes new band rejection element for implementing stop band filter. This paper also describes the synthesis, manufacture and testing of fifth-order Chebyshev filters implemented with this band rejection element.

The sixth paper "Passive frequency selective surface array as a diffuser for destroying millimeter wave coherence" proposes the design, construction, and testing of grounded frequency selective surfaces (FSS) as a diffuser for destroying millimeter wave coherence which is used to eliminate speckle in active millimeter wave imaging. The FSS arrays were designed with finite integral technique and fabricated by etching technique. The S-parameter was characterized with a free space MVNA and measurement of radiation patterns was done with a BWO in motorized setup.

In the seventh paper entitled "A new fractal-based design of stacked integrated transformers," novel fractal stacked transformers were analyzed using full-wave EM simulations and compared in terms of the inductance and quality factor. The novel fractal stacked transformers show improved performance over conventional spiral inductorbased transformers.

A novel layout of leaky-wave antennas based on tapered design has been proposed and investigated in the eighth paper "High efficiency and broadband microstrip leakywave antenna." This paper also shows that this structure is attractive for the design of high performance microstrip leaky-wave antennas for microwave and millimeter wave applications.

The ninth paper entitled "Material parameter measurements for microwave antireflection coating development" is on the material parameter measurements for microwave antireflection coating development.

Prospects of $4 \mathrm{H}-\mathrm{SiC}$ double drift region IMPATT device as a photo-sensitive high-power source at 0.7 terahertz frequency regime is presented in the tenth paper entitled 
"Prospects of $4 \mathrm{H}-\mathrm{SiC}$ double drift IMPATT device as a photo-sensitive high power source at 0.7 terahertz frequency regime."

The last paper entitled "Modeling and measurements of novel monolithic filters" presents novel miniaturized multilayer tunable high Q-filters based on hair pin resonators including ferroelectric materials and it is suitable for chip and package integration and narrow band applications.

\section{ACKNOWLEDGMENTS}

The editors are pleased to submit this special issue to Hindawi Publishing Corporation and hope that this issue accomplishes their goal of highlighting outstanding advances in the active and passive microwave devices and circuits. Thanks are expressed to all the reviewers who have read the abstracts and articles. Their contribution has been most valuable.

T. S. Kalkur

Tibor Berceli

Fahrettin Yakuphanoglu 

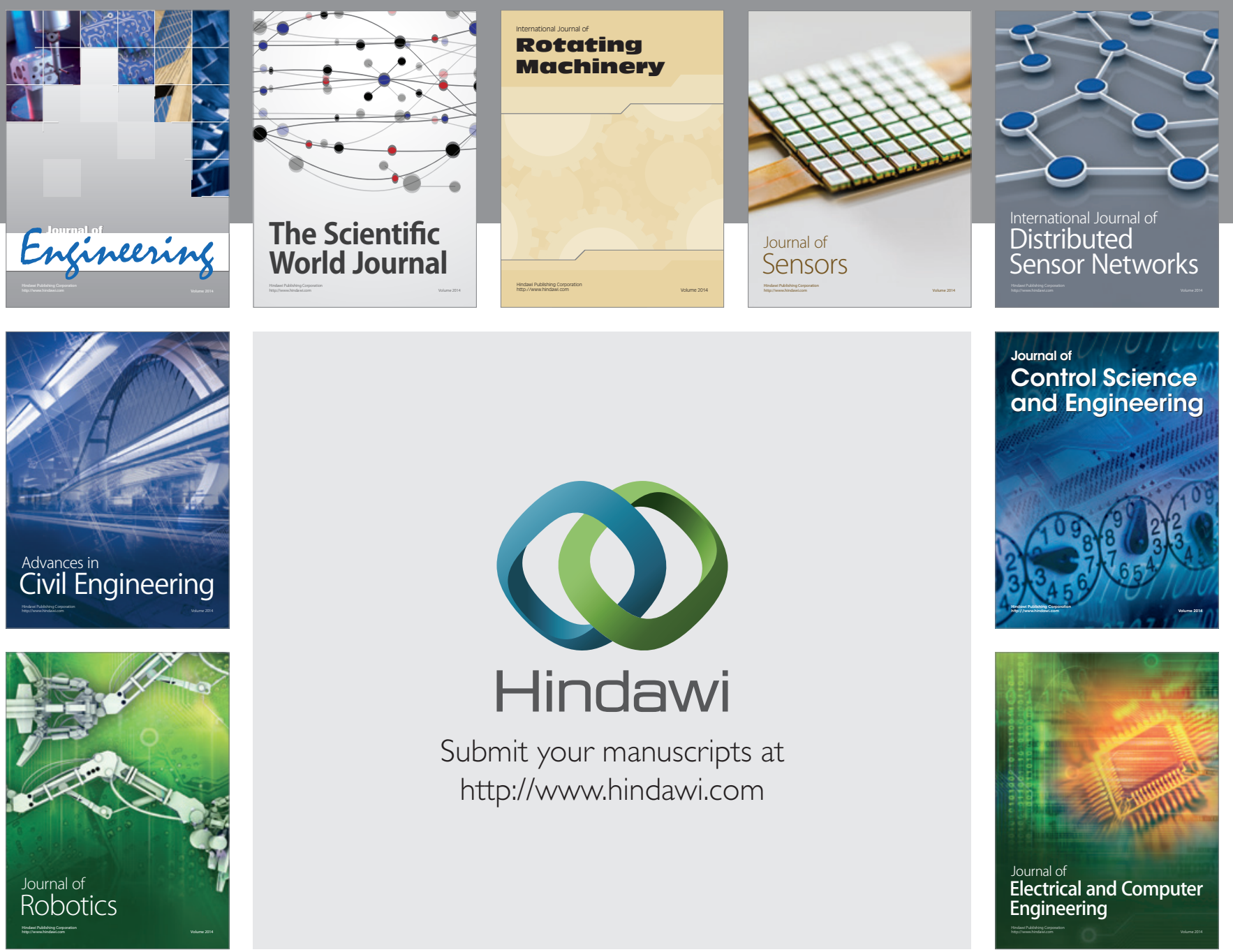

Submit your manuscripts at

http://www.hindawi.com
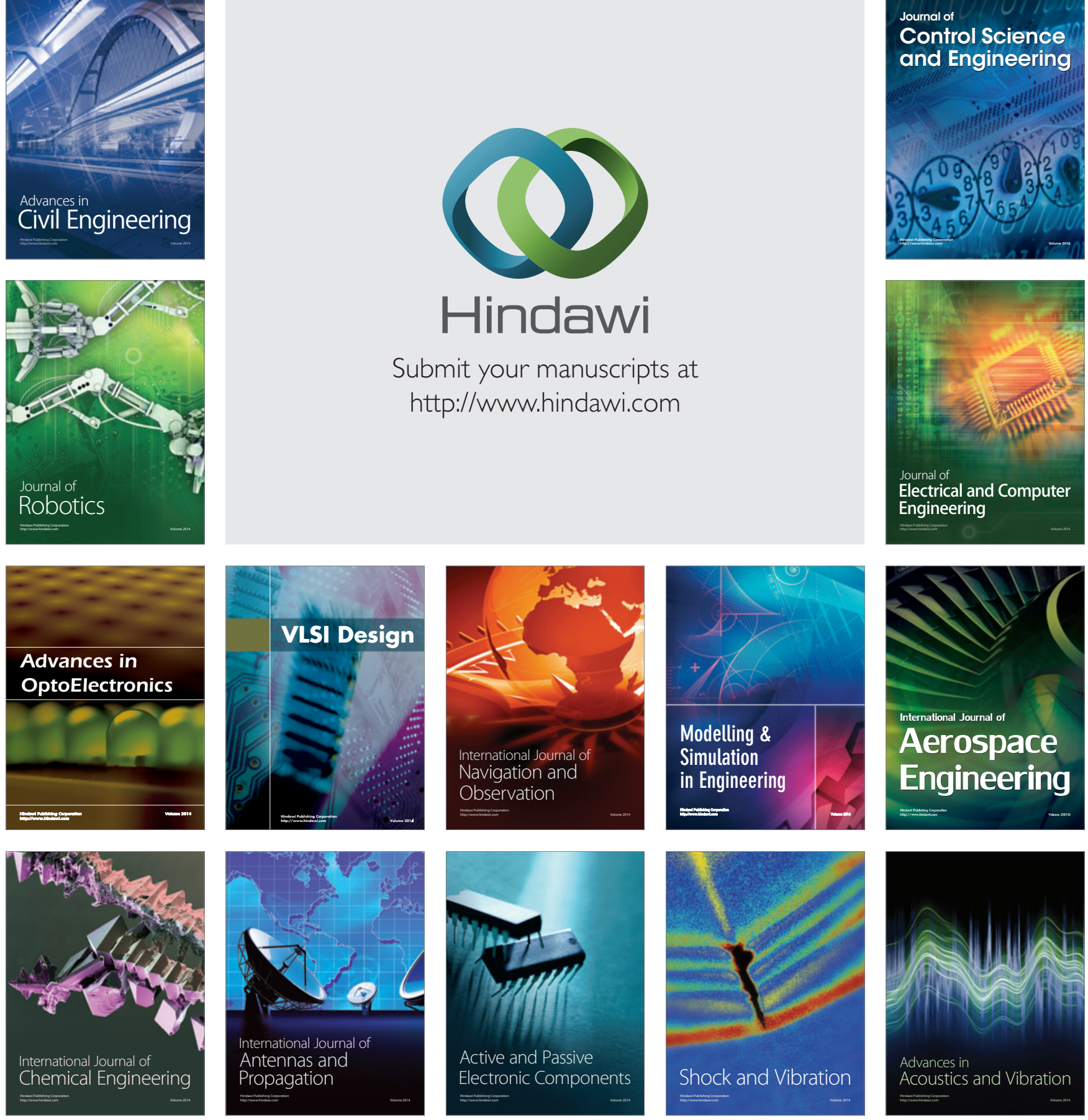